\title{
Revealing the basis of energy metabolic deficiency common to neurodegenerative diseases with differential expression meta-analysis
}

\author{
A. Osypov ${ }^{1,2 *}$, I. Yu. Popova ${ }^{2}$ \\ ${ }^{1}$ Institute of Higher Nervous Activity and Neurophysiology RAS, Moscow, Russia \\ ${ }^{2}$ Institute of Theoretical and Experimental Biophysics RAS, Pushchino MR, Russia \\ *e-mail:aosypov@gmail.com
}

Key words: neurodegenerative diseases, differential expression, meta-analysis

\begin{abstract}
Motivation and Aim: "Hypometabolism, characterized by decreased brain glucose consumption, is a common feature of many neurodegenerative diseases. Initial hypometabolic brain state, created by characteristic risk factors, may predispose the brain to acquired epilepsy and sporadic Alzheimer's and Parkinson's diseases. Deficient glucose metabolism is likely a primary initiating factor for these diseases, and resulting neuronal dysfunction further promotes the metabolic imbalance, establishing an effective positive feedback loop and a downward spiral of disease progression. Therefore, metabolic correction leading to the normalization of abnormalities in glucose metabolism may be an efficient tool to treat the neurological disorders by counteracting their primary pathological mechanisms" [1].
\end{abstract}

Methods and Algorithms: Differential expression meta-analysis.

Results: Differential expression meta-analysis reveal the proteins whose expression profiles match under all neurodegenerative diseases and differ both from that in the normal healthy stage as well as under specific diseases such as epilepsy or Alzheimer's disease. This filter out the particulars of specific neuropathologies and bring ahead the coherent groups of genes underlying the characteristics of the problem of the vicious circle of hypometabolism in neurodegenerative diseases and the consequent neuropathology itself. Future planned addition to the analysis of the expression profiles from the individuals treated with the energy supply metabolites (i.e. pyruvate) under the normal and pathological conditions will help to reveal the gene basis of the neuroprotective effects of energy metabolic correction and further elaborate the patients treatment strategy.

Another planned direction of the research is to include into the analysis the information of the individual viability to the pathological factors that may reveal the weak and strong parts of the system and the potential targets to individual treatment as well as possible common though not total resisting and labile mechanisms.

Conclusion: Here we propose a method that can be used to reveal the genetic basis of energy metabolic deficiency common to neurodegenerative diseases.

Acknowledgements: The work is supported by the fundamental research program of the Presidium of the Russian Academy of Sciences "Fundamental Research for Biomedical Technologies" for 2018.

\section{References}

1. Zilberter Y., Zilberter M. (2017) The vicious circle of hypometabolism in neurodegenerative diseases: Ways and mechanisms of metabolic correction. J Neurosci Res. DOI 10.1002/jnr.24064. 